\title{
Overview of the International Coherent Amplifying Network (ICAN)
}

\author{
William S. BROCKLESBY, ${ }^{1}$ Gerard MOUROU, ${ }^{2}$ Toshiki TAJIMA, ${ }^{3}$ and Jens LIMPERT ${ }^{4}$ \\ ${ }^{1}$ ORC, University of Southampton \\ ${ }^{2}$ Ecole Polytechnique \\ ${ }^{3}$ University of California, Irvine \\ ${ }^{4}$ Friedrich-Schiller University, Jena, Germany
}

(Received December 10, 2013)

\begin{abstract}
The ICAN project has developed a design for a high pulse energy laser which also has high average power and high efficiency. An outline of the design is given, and the potential applications for such a laser are discussed. The existence of a laser with these combined properties will hugely increase the applications feasible for petawatt lasers.
\end{abstract}

Key Words: Petawatt laser, Flash lamp pumped laser, Diode-pumped laser, Optical fiber laser, Coherent combination

\section{Introduction}

The development of real world applications for the new science emerging from petawatt lasers requires the development of laser sources that can run at high repetition rates, and simultaneously at high efficiency. Two principal issues face present day petawatt lasers: firstly, the rate at which petawatt pulses can be produced by a laser source is typically very low. The state of the art at present is the BELLA laser, ${ }^{\dagger 1}$ at Lawrence Berkeley Laboratories, which can produce 1 pulse per second. This is a major improvement over most petawatt facilities, whose pulse repetition rate is such that only a few pulses are produced per day. Any application that requires many pulses, such as an accelerator studying rare collisions, needs to study many events, and hence requires much higher pulse repetition rates.

Secondly, the petawatt sources of the present are typically based on the use of very inefficient technologies. The optical power for most PW lasers originates in flashlamps, which have very low electrical to optical conversion efficiencies. When the laser is operation at low repetition rates and hence low average powers, this low efficiency is not particularly problematic. As an example, the BELLA laser produces $\sim 40 \mathrm{~J}$ pulses at a rate of $1 \mathrm{~Hz}$, giving an average power of 40 watts the same as a typical household lightbulb. The input electrical power necessary to produce this $40 \mathrm{~W}$ output is $\sim 130 \mathrm{~kW}$, implying an efficiency of $0.03 \%$. While the pulse repetition rate is $1 \mathrm{~Hz}$ this remains possible. If we anticipate an increase of repetition rate to, for example, $10 \mathrm{kHz}$, then the input power required would be $1.3 \mathrm{GW}$, which is clearly impractical.

To get over the many issues of flashlamp-based PW lasers, groups around the world are now developing high pulse energy systems based on semiconductor diode pumped lasers, which can provide both high average powers and high efficiency. The diodes are used within a master oscillator/power amplifier configuration, and the final power amplifiers must be capable of amplifying femtosecond seed pulses from a lowpower femtosecond oscillator up to the level of tens of Joules. Pulse energies of $\sim 10 \mathrm{~J}$ at repetition rates of $\sim 10 \mathrm{kHz}$ imply average powers in the $100 \mathrm{~kW}$ region, and the resultant thermal load on the amplifiers is the key issue in laser development.

The ICAN concept uses optical fibre amplifiers to deal with the thermal issues and efficiency problems of current designs. Optical fibre amplifers based on $\mathrm{Yb}^{3+}$ are extremely efficient continuous-wave amplifiers can have efficiencies of $\sim 95 \%$. Optical fibres also provide the ideal geometry for thermal management - they have an extremely high surface/volume ratio, and heat transfer out of the lasing region can be very effective. Optical fibre lasers have been demonstrated at extremely high $\mathrm{cw}$ output powers, with the $\mathrm{kW}$ barrier having been crossed almost 10 years ago.

However, problems arise when optical fibres are used to amplify femtosecond pulses. The high peak powers involved cause both nonlinear pulse distortion and eventually damage to the fibres. In a conventional PW laser, the peak power is reduced by stretching the pulse in time using chirped pulse amplification, and also by spreading the pulse in space - i.e. working with very large (up to tens of $\mathrm{cm}$ ) beam diameters. Unfortunately, working with large beams exacerbates the thermal transport issues, as the centre of the laser material is hard to cool. Cooling of the laser material face using an optically thin coolant such as helium is being successfully used in some diode-pumped solid state systems. I) In the ICAN concept, the pulses are stretched temporally using CPA, but instead of broadening the beam in space by defocusing, the beam is instead split into many small beams, each of which is amplified in a single optical fibre. The beams for all the fibres are then recombined coherently, to form a single beam, and this can be recompressed using the usual compressor technology.

Given the present day properties of high pulse energy optical fibre amplifiers, a first-order calculation indicates that the

${ }^{\dagger 1}$ Preuss, P. BELLA Laser Achieves World Record Power at One Pulse Per Second. at <http://newscenter.lbl.gov/news-releases/2012/07/27/bellalaser-record-power/> 
creation of pulses in the PW power regime will require thousands of fibre amplifiers to be recombined into a single beam. The credibility of the ICAN concept rests on the feasibility of producing a laser system that combines thousands of fibre lasers coherently, for a reasonable cost and in a reasonable time. The present ICAN project has been dedicated to answering this question, and the answer is a definite 'yes'.

\section{The ICAN laser design}

Several key technologies are required for the ICAN laser to work. Fig. 1 shows a block diagram of a potential ICAN system. The initial seed pulse is created using standard technology, and then stretched using standard grating or fibre based techniques. After stretching, the pulse must be split into many channels for amplification. The technology of amplifying and splitting to create the requisite number of channel can be very closely related to the technology of optical fibre communication networks, which is well-developed and thus low risk. Once sufficient channel numbers have been created by a cascade of splitters and low power amplifiers, then each individual channel must be amplified using high power amplifiers to create the final pulse for beam combination. This stage of the amplification chain is less technologically advanced, and will require development, but amplification has been demonstrated in the laboratory to the required pulse energy levels, so the technological risk here is also low.

After amplification, the beams for many thousands of lasers must be coherently combined. The coherent combination of beams on such a large scale has never been demonstrated however, several recent pieces of work suggest that combination of large numbers of beams is entirely feasible. The state of the art of beam combination for ultrafast pulsed fibre lasers is for small numbers, but is already impressive in terms of both peak and average power. Coherent combination of two pulses has demonstrated ${ }^{2)}$ pulse energies of $3 \mathrm{~mJ}$, and coherent combination of 4 pulses from fibre amplifiers ${ }^{3)}$ has produce $1.3 \mathrm{~mJ}$ pulses at an average power of $530 \mathrm{~W}$.

Coherent combination of thousands of beams requires a more scalable approach. In order to combine many thousands of pulses with interferometric accuracy, techniques have been developed which measure the relative phases of a large array of beams in a single measurement. The measurement is based on 4-way lateral shearing interferometry. The beams pass through a two-dimensional diffraction grating. Each beam produces diffraction orders that overlap with those of its 4 neighbours, and the overlapping regions are imaged onto a single array detector. By studying the interference patterns in the image, relative phases can be deduced and this information used to coherently phase the beams. This technique, know as quadriwave laterial shearing interferometry, has already been demonstrated using an array of 64 fibres. ${ }^{4}$ Recent analysis of the expected signal to noise has shown that a minimum of 8 image pixels per fibre is needed to measure the phase error distribution and allow phase locking of the system with a residual error of $\lambda / 20$. Thus phase measurement and correction for 10,000 beams would require a camera with $800 \times 800$ pixels. Calculation of the phase errors for this number of beams could be performed using a single GPU at a $1 \mathrm{kHz}$ repetition rate, high enough to remove thermal and acoustic fluctuations, which have been shown to be the dominant noise contributions. ${ }^{5}$ As for the effect of large channel numbers on coupling efficiency, analysis of the scaling of the fluctuations in systems with thousands of channels ${ }^{6}$ ) has shown that achieving $>90 \%$ combination efficiency in very large numbers of channels requires phase control to only $\lambda / 20$, and increases in phase and amplitude noise between channels causes a decrease of coupling efficiency which saturates above $\sim 100$ channels at values greater than $80 \%$ for even high levels of phase noise. Thus, phase locking of very large number of amplifiers with high efficiency and low noise is feasible with current techniques.

The thermal load within the amplifier array at high average power will be significant, although the comparison with other technologies is interesting. A $0.5 \mathrm{MW}$ fibre amplifier array operating at $67 \%$ efficiency ${ }^{7)}$ produces $\sim 250 \mathrm{~kW}$ of waste heat. This is about equivalent to the waste heat produced by a small family car engine, and two or three times the heat load produced by a large argon ion laser, which until recently was common in many research labs. The geometry of the fibre array makes thermal engineering much more flexible that for an $\mathrm{Ar}^{+}$laser. Hence although cooling will require careful engineering, solutions will be possible.

After beam combination, the temporally-stretched beam must be recompressed. This is accomplished in present PW systems using a grating-based compressor. A similar technique is feasible for an ICAN system - however, thermal design of the compressor elements needs to be done carefully in order that the compressor will be able to handle the very high average powers available. Present gratings may be capable of handling many tens of $\mathrm{kW}$ without change - however, handling hundreds of $\mathrm{kW}$ may require changes in the grating substrates or materials.

Thus each element of the ICAN concept is feasible - however, we also require that the cost of such a system is reasonable. Given that the performance of an ICAN system may be $\sim 10,000$ time that of a present PW system, the cost will not be small - a factor of ten more than present PW lasers. However, it is important to understand how the cost is distributed across the system elements, and also how the system will be manufactured - it is outside the capability of most research labs to manufacture 10,000 fibre amplifiers, and will require industry

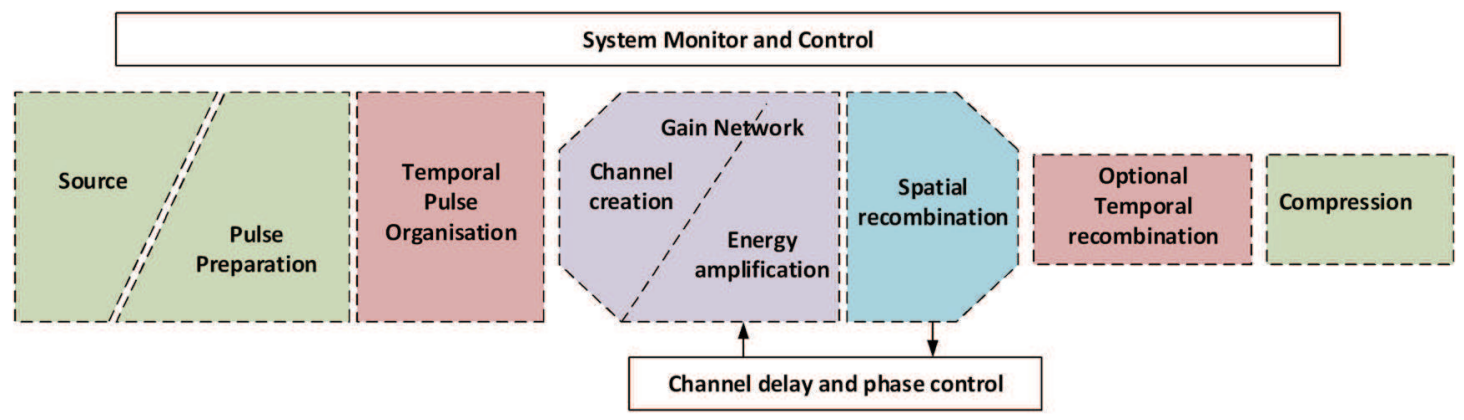

Fig. 1 Block diagram of an ICAN laser system. 
cooperation. The ICAN consortium has included many fibre manufacturers from the outset, so that the eventual system design is manufacturable, and reliability and serviceability will be engineered in from the start.

\section{Costing and manufacture}

From cost analysis, it is clear that the dominant cost within the system is the cost of the final amplifier in each channel. Thus any technique that reduces the number of channels will significantly reduce costs. Two techniques have been put forward by which channel numbers may be reduced significantly. These are known as divided pulse amplification (DPA) and pulse stacking.

Divided pulse amplification makes use of the fact that a fs pulse stretched to a few ns does not use up all of the available gain from a fibre amplifier. In order to use all the gain, a train of 4 or 8 pulse replicas separated by $\sim 10 \mathrm{~ns}$ is created using bulk optics just after the stretcher. This effectively stretches the pulse much further than conventional CPA. DPA has been discussed $^{8)}$ as a route to production of an increase in average power out of a fibre amplifier from a few $\mathrm{mJ}$ to $\sim 50 \mathrm{~mJ}$, which could reduce the number of amplifiers necessary (and, to first order, the cost) by almost an order of magnitude.

Pulse stacking uses a different technique to reduce the numbers of amplifiers necessary, and make use of the fact that optical fibre amplifiers can operate at high repetition rate more easily that they can operate at high pulse energy. In a pulse stacking system, the amplifying network is run at a high repetition rate, say $10 \mathrm{MHz}$. The pulses produced are then coupled into a $10 \mathrm{MHz}$ optical cavity, so that the pulse energies are 'stacked' and the intracavity pulse has an energy which may be 5-600 times the input pulse. After pulse buildup, the energetic pulse is switched out of the cavity at a low repetition rate, so that the $10 \mathrm{MHz}$ train is converted into a $15 \mathrm{kHz}$ pulse train with significantly higher pulse energy. High average power cavities have already been demonstrated, ${ }^{9)}$ but switch- ing the pulse out of the cavity is technologically difficult. Both of these approaches are the subject of present research by groups within the ICAN consortium, and may radically reduce the eventual system cost.

\section{Laser properties}

Apart from the originally specified parameters of high average power and high peak power, several other advantages arise from the ICAN design. Control of the phase of each individual element making up the laser beam means that it may be possible (within the constraints of temporal compression) to shape the amplitude and phase of the beam to suit the application - whether that be focusing, beam steering, or changing the beam profile. In addition, the fact that the final beam is made up of the sum of thousands of smaller beams has a significant effect on the noise properties of the pulses. Present PW laser systems can show appreciable amplitude noise across the beam, and also significant shot-to-shot variation. Simulations of the ICAN system indicate that its amplitude and shot-to-shot noise will be orders of magnitude smaller than today's lasers, which will improve the quality of results from experiments by a large degree.

\section{Applications}

The impact of the ICAN project centres on taking the applications for PW lasers from lab to user. Some of the applications are predominantly science-based, for example the production of new compact accelerators and free electron lasers, or the search for dark matter. However, many applications have potentially huge societal impact, particular in areas that can be grouped under 'nuclear photonics' - even the processing of nuclear waste starts to become viable if efficient proton acceleration can be demonstrated. In this section, we will discuss the applications and give an overview of how they can be useful.

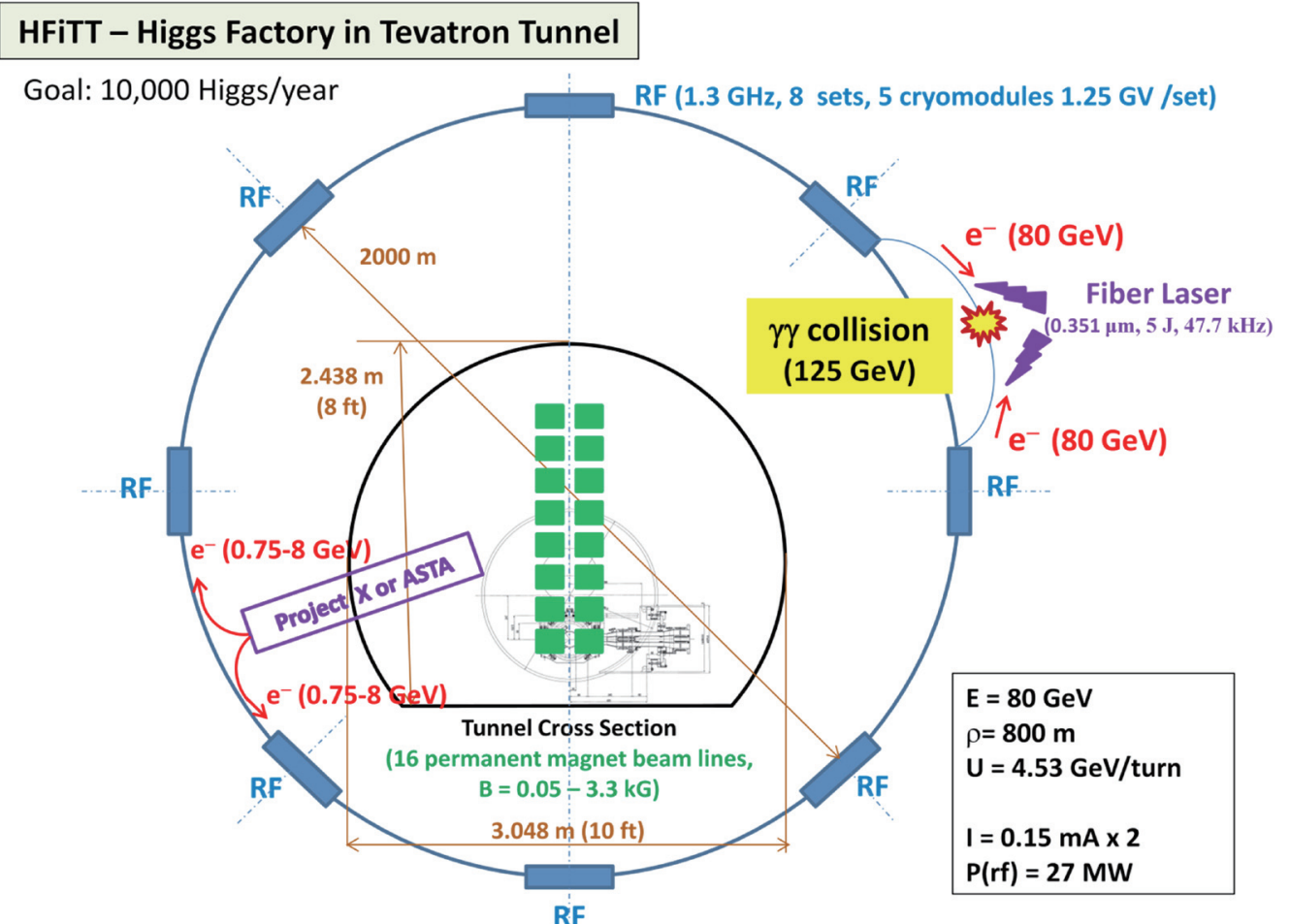

Fig. 2 The gamma-gamma colider "HFiTT" based on the ICAN laser (from reference 11). 
Immediate applications for an ICAN source include laser wake field acceleration for compact accelerators ${ }^{10)}$ and free electron lasers, $\gamma$-ray generation for Higgs factories ${ }^{11)}$ and $\mathrm{Nu}-$ clear Resonance Fluorescence imaging, ${ }^{12}$ ) and ion acceleration for accelerator-driven systems (ADS), ${ }^{13)}$ nuclear pharmacology, ${ }^{\dagger 2}$ and proton therapy. ${ }^{\dagger 2}$ Applications in dark matter detec$\operatorname{tion}^{14)}$ using photon-photon interactions are also being pursued.

These applications can be divided into groups based on their underlying physics mechanisms - LWFA is based around plasma wave generation; NRF and $\gamma \gamma$ colliders are based on photon-electron scattering, and ADS, nuclear pharmacology, and proton therapy are based on relativistic ion acceleration. Each requires a different level of laser development, and each will represent steps along the way as the ICAN laser power increases.

\section{Wake field acceleration}

The amplitude of the plasma wake field created by a laser pulse is proportional to the peak intensity of the laser pulse. Higher wake field amplitudes are equivalent to larger voltages accelerating the free electrons, which implies the same velocities can be reached within shorter distances. This results in a more compact system.

To achieve high pulse peak intensities, the pulse energy should be as high as possible, the duration as short as possible and the beam quality as good as possible (more details in the following). According to the joint ICFA-ICUIL task force on lasers for accelerators, ${ }^{15)} 32 \mathrm{~J}$ of pulse-energy in a pulse with the duration of sub $300 \mathrm{fs}$ and a repetition rate of $15 \mathrm{kHz}$, would be sufficient for each stage of an accelerator which might consist of 100 stages of $1 \mathrm{GeV}$ acceleration - these were the parameters taken as the starting point for the ICAN project. A compact accelerator producing 1-10 GeV energy electrons would also be a good candidate for a compact FEL system - the FLASH laser at DESY only uses $1.25 \mathrm{GeV}$ electrons, and LCLS in the region of $10 \mathrm{GeV}$.

\section{Electron-photon scattering}

Two examples of the use of a combination of an electron beam and a high intensity photon source in applications are immediately apparent for the ICAN laser. Both use the laser to produce $\gamma$ radiation.

The ICAN concept has reinvigorated the possibility of Higgs factories based on photon colliders. Producing Higgs particles by collisions between photons and electrons puts the lowest requirements on electron beam energy, and as such is an attractive option. It is proposed that the Tevatron tunnel at Fermilab could be re-used to provide the storage ring for such an electron-photon collider ${ }^{11)}$ (HFiTT, "Higgs factory in the Tevatron tunnel"). Two counter-propagating beams collide with photons from two laser systems, and the resultant $\gamma$ emission produces $\gamma \gamma$ collisions which can produce the Higgs particles. The target flux of Higgs for the Tevatron-based collider is $\sim 10 \mathrm{k}$ Higgs/year.

The laser requirement for a $\gamma \gamma$ collider is slightly different from that for a laser wake field accelerator - the peak power needed is lower, but the repetition rate is higher, and the wavelength is much shorter. In general, lower pulse energy and higher repetition rate is beneficial for fibre laser based systems, as the high peak power is more problematic than the high average power. The laser specification needs to match the present accelerator repetition rate of $47.7 \mathrm{kHz}$, at pulse energies of $\sim 5 \mathrm{~J}-$ an average power of $240 \mathrm{~kW}$. Although still way beyond the reach of present laser systems, these parameters are within the design spec of the ICAN laser.

Generation of $\gamma$ emission via laser Compton scattering is also the basis for nuclear resonance fluorescence imaging, which has potential uses in identifying, for example, shielded chemical compounds within containers. Detection of organics within steel and lead containers has been demonstrated using conventional $\gamma$ sources. ${ }^{16)}$ The development of a compact, efficient $\gamma$ source based on an ICAN laser could have applications in security, and in non-contact identification of nuclear waste materials, for example the kind of waste produce in the recent Fukushima incident.

\section{Relativistic Ion Acceleration}

Relativistic ion acceleration requires laser intensities that are several orders of magnitude higher than those required for electron acceleration. Several mechanisms have been proposed for acceleration at these energies; the most exciting is Radiation Pressure Acceleration ${ }^{17)}$ (RPA), in which the ions produced can be monoenergetic, and coupling from radiation to ions is predicted to be very efficient. If RPA can be demonstrated using an ICAN source, then the way is opened to efficient relativistic ion generation, which has immediate applications in ion therapy, ${ }^{\dagger 2}$ and even more radical applications in potential laser-driven spallation neutron sources. Neutron sources are key to the development of accelerator-driven systems (ADS) and accelerator-driven reactors (ADR), technologies which may revolutionize the area of nuclear power. ADRs can provide inherently safe reactors, in which chain reactions are not possible and the nuclear reaction is driven by an external neutron source - if the neutron source is stopped, the reaction stops. ADS can also provide a means of reducing nuclear waste, as the controllability and safety of an ADR means that it can be used to 'burn' the minor actinides which make up the most difficult to handle and dangerous of much nuclear waste, converting it from material with half-life of hundreds of thousands of years to material with half-life of tens of years, which is inherently much easier to store and dispose of. An ICAN laser could drive a proton accelerator, which can be used to create a laser-driven spallation neutron source - a schematic is shown in Fig. 3.

\section{Summary}

The ICAN project so far has demonstrated the route to construction of an efficient high average power petawatt laser source. The physical and engineering constraints have been examined in detail, and a route to a design that is both feasible and relatively affordable has been identified. As the project has proceeded, new applications have been found with important scientific and societal implications, and it is likely that many more will be found as the ICAN technology model becomes widespread.

\footnotetext{
${ }^{\dagger 2}$ Dietrich Habs, Toshiki Tajima, and Ulli Köster (2011). Laser-Driven Radiation Therapy, Current Cancer Treatment - Novel Beyond Conventional Approaches, Prof. Oner Ozdemir (Ed.), ISBN: 978-953-307-397-2, InTech, DOI: 10.5772/24190. Available from: http://www.intechopen.com/ books/current-cancer-treatment-novel-beyond-conventional-approaches/laser-driven-radiation-therapy
} 


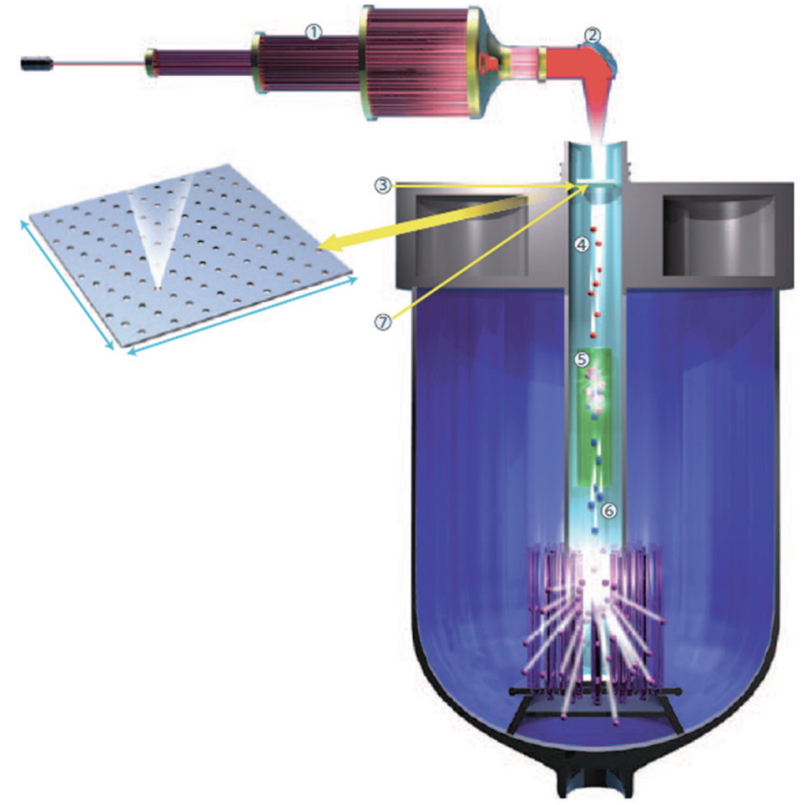

Fig. 3 Schematic of laser-driven spallation neutron source for ADS (from reference 13).

\section{References}

1) K. Ertel et al.: DiPOLE: a scalable laser architecture for pumping multi-Hz PW systems. in (Hein, J., Korn, G. \& Silva, L. O.) 87801W-87801W-5 (2013). doi:10.1117/12.2021330.

2) A. Klenke et al.: Opt. Express 19 (2011) 24280.

3) A. Klenke et al.: Opt. Lett. 38 (2013) 2283

4) J. Bourderionnet, C. Bellanger, J. Primot, and A. Brignon: Opt. Express. 19 (2011) 18

5) C. Bellanger et al:: Opt. Lett. 35 (2010) 3931.

6) L. A. Siiman, W. Chang, T. Zhou, and A. Galvanauskas: Opt. Express 20 (2012) 18097

7) T. Eidam et al.: Opt. Lett. 35 (2010) 94.

8) M. Kienel et al.: Opt. Express 21 (2013) 29031.

9) I. Pupeza et al.: Opt. Lett. 35 (2010) 2052.

10) W. Leemans and E. Esarey: Phys. Today 62 (2009) 44.

11) W. Chou, G. Mourou, N. Solyak, T. Tajima, and M. Velasco: "HFiTT - Higgs Factory in Tevatron Tunnel" Fermilab-TM-2558APC (2013).

12) T. Hayakawa et al.: Rev. Sci. Instrum. 80 (2009) 045110.

13) G. Mourou, B. Brocklesby, T. Tajima, and J. Limpert: Nat. Photonics 7 (2013) 258

14) K. Homma, D. Habs, and T. Tajima: Appl. Phys. B 106 (2011) 229.

15) W. Leemans: ICFA Beam Dyn. Newslett. 56 (2011) 10.

16) T. Hayakawa et al.: Rev. Sci. Instrum. 80 (2009) 045110.

17) T. Esirkepov, M. Borghesi, S. Bulanov, G. Mourou, and T. Tajima: Phys. Rev. Lett. 92 (2004) 175003. 